\title{
DETERMINING REACTION FORCES IN PLANAR MECHANISMS
}

\author{
Do Sanh ${ }^{1}$, Dinh Van Phong ${ }^{1}$, Do Dang Khoa ${ }^{1}$, Phan Dang Phong ${ }^{2}$ \\ ${ }^{1}$ Hanoi University of Technology, \\ ${ }^{2}$ National Research Institute of Mechanical Engineering, Hanoi
}

\begin{abstract}
In the paper, it is introduced a method to determine joint reaction forces, constraint forces and internal forces at the cross section of linkages. Based on the principle of compatibility and the ideality of constraints, the methodology is presented to analyze and determine reaction forces in planar mechanisms.
\end{abstract}

\section{INTRODUCTION}

There are two main objectives in Dynamics. One is to determine the motion of dynamic systems, and the other is to specify forces exerted on that system. Determining reaction forces is part of the latter. It is important not only to the dynamics analysis of systems and its endurance but also to its control problems, especially the program motion one. In the program motion problem, reaction forces are considered as control inputs whereas constraints can be understood as the program to be realized. Generally, reaction forces are determined based on d'Alembert principle by solving the equations of dynamic equilibrium. The principle reduces the problem of dynamics to a problem in statics by adding the forces of inertia. The forces of inertia combine with the externally applied forces to produce dynamic equilibrium. However, this approach is not always easy to apply for complicated systems, especially for mechanisms. In the paper, another approach to determine reaction forces is presented based on the principle of compatibility and the ideality of constraints. It is a matrix-based approach so that one can easily use common software such as Mathlab, Maple, and MathCad to assist the calculation process.

\section{BACKGROUND}

Let's consider a dynamic system whose positions are located by the generalized coordinates $\mathrm{q}_{i}(i=\overline{1, m})$. It assumes that all the constraints are stationary and ideal. The kinetic energy of the whole system has the form

$$
T=\frac{1}{2} \sum_{i, j=1}^{m} a_{i j} \dot{q}_{i} \dot{q}_{j},
$$


where $a_{i j}$ is a function of the generalized coordinates $q_{i}(i=\overline{1, m})$. Equation (1) can be expressed in the matrix form as follows.

$$
T=\frac{1}{2} \dot{\mathbf{q}}^{\mathbf{T}} \mathbf{A} \dot{\mathbf{q}}
$$

where $\dot{q}$ is a column vector of $m \times 1$ size of generalized velocities, $\mathbf{A}$ is a matrix of inertiaa square symmetric nonsingular one, $\dot{\mathbf{q}}^{T}$ is the transpose of $\dot{\mathbf{q}}$.

Let's define the generalized forces correcponding to the generalized coordinates as $Q_{i}\left(i=\overline{1, m)}\right.$ or in the vector form as $\mathbf{Q}^{T}=\left\|Q_{1} \quad Q_{2} \quad . \quad Q_{m}\right\|$. The dynamic system is subject to $\mathrm{r}$ constraints as follows

$$
f_{\alpha}\left(q_{1}, q_{2}, \ldots, q_{m}\right)=0 ; \quad \alpha=\overline{1, r} .
$$

Based on the principle of compatibility $[1,2]$, the equations of motion of the dynamic system are given by

$$
\frac{d}{d t} \frac{\partial T}{\partial \dot{q}_{i}}-\frac{\partial T}{\partial q_{i}}=Q_{i}+R_{i} ; \quad i=\overline{1, m}
$$

where $R_{i}(\mathrm{i}=\overline{1, \mathrm{~m}})$ are the generalized forces of reaction forces in the constraints $(3)$ corresponding to the generalized coordinates $q_{i}(i=\overline{1, m})$

$$
R_{i}=\sum_{k=1}^{N} \vec{N}_{k} \frac{\partial \vec{r}_{k}}{\partial q_{i}}
$$

where $\vec{N}_{k}$ are reaction forces of the constraints exerted on a point mass $\mathrm{M}_{k}$ of the system. Equation (4) can be expressed in the matrix form as

$$
\frac{d}{d t} \frac{\partial T}{\partial \dot{\mathbf{q}}}-\frac{\partial T}{\partial \mathbf{q}}=\mathbf{Q}+\mathbf{R}
$$

where $\mathbf{R}$ is a column vector of $m \times 1$ size whose elements are the generalized forces of reaction forces. The constraints (3) are assumed ideal so that the generalized forces of reaction forces $R_{i}(i=\overline{1, m})$ must realize the condition $[1,2]$

$$
\sum_{i=1}^{m} d_{i k} R_{i}=0 ; \quad k=\overline{1, n}
$$

where $d_{i k}(i=\overline{1, m} ; k=\overline{1, n})$ are elements of transformation matrix which maps the independent generalixed accelerations $\ddot{q}_{k}(k=\overline{1, n})$ into the generalized accelerations $\ddot{q}_{i}(i=$ $\overline{1, m)}$. Expression $(7)$ can be given in the matrix form as

$$
\mathrm{DR}=\mathbf{0},
$$

where $\mathbf{D}=\left\|d_{k i}\right\|$, the matrix of $n \times m$ size. Equation (6) can be rewritten in a new form as

$$
\mathbf{A} \ddot{\mathbf{q}}=\mathbf{Q}+\mathbf{Q}^{0}-\mathbf{Q}^{\mathbf{g}}+\mathbf{R},
$$

where $\mathbf{Q}^{0}, \mathbf{Q}^{\mathbf{g}}$ are determined through the matrix of inertia $\mathbf{A}[6]$. Given on (8), and (9), the reaction forces of constraints of the system are specified. 


\section{TWO PROBLEMS OF DETERMING REACTION FORCES}

\subsection{Problem 1}

Assuming that the dynamic system is subject to the constraints of the form

$$
q_{\alpha}=0, \quad(\alpha=\overline{1, r}=m-n) .
$$

It means that some generalized coordinates of the system are constrained and the system's order of freedom is $n$. For convenience, let's define some new variables as

$$
u_{\dot{k}} \equiv q_{k}(k=\overline{1, n}) ; v_{\alpha} \equiv q_{\alpha}(\alpha=\overline{1, r}=m-n) .
$$

Based on (11), (9) can be expressed

$$
\left\|\begin{array}{l}
\mathbf{R}(\mathbf{k}) \\
\mathbf{R}(\alpha)
\end{array}\right\|=\left\|\begin{array}{cc}
\mathbf{A}_{1} & \mathbf{A}_{\mathbf{3}} \\
\mathbf{A}_{3}^{\mathrm{T}} & \mathbf{A}_{\mathbf{2}}
\end{array}\right\|\left\|\begin{array}{l}
\ddot{\mathbf{u}}(\mathbf{k}) \\
\ddot{\mathbf{v}}(\alpha)
\end{array}\right\|+\left\|\begin{array}{l}
\mathbf{Q}(\mathbf{k}) \\
\mathbf{Q}(\alpha)
\end{array}\right\|+\left\|\begin{array}{c}
\mathbf{Q}^{\mathbf{0}}(\mathbf{k}) \\
\mathbf{Q}^{\mathbf{0}}(\alpha)
\end{array}\right\|-\left\|\begin{array}{c}
\mathbf{Q}^{\mathbf{g}}(\mathbf{k}) \\
\mathbf{Q}^{\mathbf{g}}(\alpha)
\end{array}\right\|,
$$

where: $\mathbf{R}(\mathbf{k}), \ddot{\mathbf{u}}(\mathbf{k}), \mathbf{Q}(\mathbf{k}), \mathbf{Q}^{\mathbf{0}}(\mathbf{k}), \mathbf{Q}^{\mathbf{g}}(\mathbf{k})$ are column vectors of nx1 size, but $\mathbf{R}(\alpha), \ddot{\mathbf{v}}(\alpha), \mathbf{Q}(\alpha)$, $\mathbf{Q}^{\mathbf{0}}(\alpha), \mathbf{Q}^{\mathbf{g}}(\alpha)$ are ones of $r \times 1$ size.

$$
\mathbf{R}(k)=\left\|\begin{array}{llll}
R_{1} & R_{2} & R_{n}
\end{array}\right\| ; \quad \mathbf{R}(\alpha)=\left\|R_{n+1} \quad R_{n+2} \quad R_{m}\right\| .
$$

Due to (10), the matrix $\mathbf{D}$ has the form $\mathbf{D}=\|\mathbf{D}(k) \mathbf{D}(\alpha)\|$, where $\mathbf{D}(\mathrm{k})$ is a square matrix of $n$ size as

$$
\mathbf{D}(k)=\left\|\begin{array}{cccc}
1 & 0 & 0 & 0 \\
0 & 1 & 0 & 0 \\
0 & 0 & 1 & 0 \\
0 & 0 & 0 & 1
\end{array}\right\|
$$

and $\mathbf{D}(\alpha)$ is a zero matrix of nxr size.

The condition of ideality (8) can be written in a new form as

$$
\|\mathbf{D}(\mathbf{k}) \quad \mathbf{D}(\alpha)\|\left\|\begin{array}{l}
\mathbf{R}(\mathbf{k}) \\
\mathbf{R}(\alpha)
\end{array}\right\|=0
$$

Obviously, given on (15), one realizes

$$
R_{k}=0 ; \quad k=\overline{1, n} .
$$

In this case, some conditions are applied as

$$
v_{\alpha}=\dot{v}_{\alpha}=\ddot{v}_{\alpha}=0 ; \alpha=\overline{1, r}=m-n ; u_{k}=q_{k} ; \dot{u}_{k}=\dot{q}_{k} ; \ddot{u}_{k}=\ddot{q}_{k} ; k=\overline{1, n} .
$$

Based on (12), one can derive that

$$
\mathbf{R}(\alpha)=\mathbf{A}_{3}^{T} \ddot{\mathbf{u}}(\mathbf{k})+\mathbf{Q}(\alpha)+\mathbf{Q}^{\mathbf{0}}(\alpha)-\mathbf{Q}(\alpha)^{\mathbf{g}},
$$

where $\ddot{\mathbf{u}}^{T}(k) \equiv\left\|\ddot{q}_{1} \ddot{q}_{2} \ldots \ddot{q}_{n}\right\|$ which can be determined through

$$
\mathbf{A}_{\mathbf{1}} \ddot{\mathbf{u}}=\mathbf{Q}(\mathbf{k})+\mathbf{Q}^{0}(\mathbf{k})-\mathbf{Q}^{\mathrm{g}}(\mathbf{k}) .
$$

Through this approach, reaction forces of (10) are specified by (18) and (19). 


\subsection{Problem 2}

Assume that the dynamic system is subject to the constraints (3). Let's apply the mapping from the current coordinates $\left(q_{i}, i=\overline{1, m}\right)$ into the new coordinates $\left(u_{k}, v_{\alpha}\right)$ as

$$
u_{k} \equiv q_{k}(k=\overline{1, n}) ; v_{\alpha} \equiv q_{\alpha}(\alpha=\overline{1, r}=m-n) .
$$

Let's define $\mathbf{K}$ as a transformation matrix from the current coordinates to the new coordinates. The kinetic energy of the system in the new coordinates now has the form (2) in which the matrix of inertia $\overline{\mathbf{A}}$ is defined as

$$
\overline{\mathbf{A}}=\mathbf{K}^{\mathbf{T}} \mathbf{A}\left(\mathbf{u}_{k}, \mathbf{v}_{\alpha}\right) \mathbf{K} .
$$

The generalized forces $\mathbf{Q}$ has the form

$$
\overline{\mathbf{Q}}=\mathbf{K}^{\mathbf{T}} \mathbf{Q} \text {. }
$$

By doing that, the problem 2 can be converted into the problem 1 where $\overline{\mathbf{A}}$ and $\overline{\mathbf{Q}}$ take place of $\mathbf{A}$ and $\mathbf{Q}$, respectively. In this case, let's notice the conditions as

$$
u_{k} \equiv q_{k} ; \dot{u}_{k} \equiv \dot{q}_{k} ; \ddot{u}_{k} \equiv \ddot{q}_{k} ; v_{\alpha} \equiv 0 ; \dot{v}_{\alpha} \equiv 0 ; \ddot{v}_{\alpha} \equiv 0 .
$$

The following section illustrates the two above approaches by some simple examples.

\section{ILLUSTRATIVE EXAMPLES}

\subsection{Example 1}

Determine the reaction forces between joint A of the double pendulum OAB. Link $\mathrm{OA}$ of length $\mathrm{L}_{1}$, of mass $\mathrm{m}_{1}$, pivots about the fixed point $\mathrm{O}$. Its center of mass locates at $C_{1}$ and moment of inertia to $C_{1}$ is $J_{1}$. Link $A B$ of length $L_{2}$, of mass $m_{2}$ rotates about the joint $A$. Its center of mass locates at $\mathrm{C}_{2}$ and the moment of inertia to $\mathrm{C}_{2}$ is $\mathrm{J}_{2}$. The motion of the dynamic system is analyzed in the field of conservative forces. From now on, some notations are used for convenience

$$
\cos \varphi_{i} \equiv C_{i} ; \quad \sin \varphi_{i} \equiv S_{i} ; \quad \cos \left(\varphi_{i}+\varphi_{j}\right) \equiv C_{i j} ; \quad \sin \left(\varphi_{i}+\varphi_{j}\right) \equiv S_{i j} .
$$

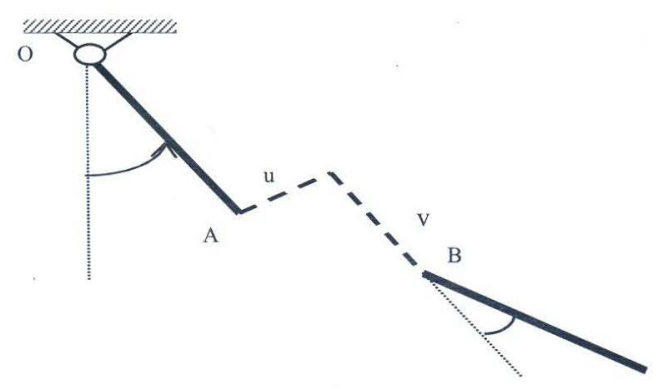

Fig. 1. The internal forces at the cross section

The order of freedom of the system is 2 . Choose the generalized coordinates are $\varphi_{1}$ and $\varphi_{2}$, where $\varphi_{1}$ is the inclined angle between link $\mathrm{OA}$ and the vertical, $\varphi_{2}$ is the angle between link $\mathrm{OA}$ and link $\mathrm{AB}$, see Fig. 1. This system is considered as the origin one. 
In Fig. 1, joint $\mathrm{A}$ is released and two more coordinates $(u, v)$ are used to describe the system's position. The number of coordinates of the new system is now 4. Let's apply the following conditions to make the new system coincide with the previous one.

$$
f_{1} \equiv u=0 ; \quad f_{2} \equiv v=0
$$

Finding the matrix of inertia $\mathbf{A}$ of the constraint-released system can be carried out through the method of transmission matrix $[4,5]$ or the direct approach. The matrix $\mathbf{A}$ of $4 \times 4$ sizes is given as follows

$$
\begin{aligned}
& a_{11}=m_{2}\left(L_{1}^{2}+u^{2}+v^{2}+a_{2}^{2}+2 L_{1} a_{2} C_{2}+2 L_{1} v+2 a_{2} S_{2} u+2 a_{2} C_{2} v\right)+J_{1}+J_{2} ; \\
& a_{12}=m\left(L_{1} C_{2}+a_{2}+u S_{2}+v C_{2}\right) a_{2} ; a_{22}=J_{2}+m_{2} a_{2}^{2} ; a_{1 u}=m_{2}\left(L_{1}+a_{2} C_{2}+v\right) ; \\
& a_{2 u}=m_{2} a_{2} C_{2} ; a_{u u}=m_{2} ; a_{1 v}=-m_{2}\left(a_{2} S_{2}+u\right) ; a_{2 v}=-m_{2} a_{2} S_{2} ; a_{v u}=0 ; a_{v v}=m .
\end{aligned}
$$

The potential energy has the form

$$
\pi=-m_{1} g a_{1} C_{1}-m_{2} g\left(L_{1} C_{1}-u S_{1}+v C_{1}+a_{2} C_{12}\right) .
$$

The matrix $\mathbf{Q}$ of generalized forces is of the form

$$
\mathbf{Q}=\left\|\begin{array}{c}
-m_{1} g a_{1} S_{1}-m_{2} g\left(L_{1} S_{1}-u C_{1}+v S_{1}+a_{2} S_{12}\right. \\
-m_{2} g a_{2} S_{12} \\
-m_{2}^{g} S_{1} \\
m_{2} g C_{1}
\end{array}\right\|
$$

The matrix $\mathbf{Q}^{o}$ and $\mathbf{Q}^{g}$ can be derived

$$
\mathbf{Q}^{0}=\left\|\begin{array}{l}
0 \\
-m_{28} L_{1} a_{2} S_{2}\left(\dot{\varphi}_{1}+\dot{\varphi}_{2}\right) \dot{\varphi}_{1} \\
m_{2} a_{2} S_{2}\left(\dot{\varphi}_{1}+\dot{\varphi}_{2}\right) \dot{\varphi}_{1} \\
m_{2}\left(L_{1}+2 a_{2} C_{2}\right)\left(\dot{\varphi}_{1}+\dot{\varphi}_{2}\right) \dot{\varphi}_{1}
\end{array}\right\| ; \quad \mathbf{Q}^{g}=\left\|\begin{array}{l}
-m_{2} L_{1} a_{2} S_{2}\left(2 \dot{\varphi}_{1}+\dot{\varphi}_{2}\right) \dot{\varphi}_{1} \\
m_{2} L_{1} S_{2} a_{2} \dot{\varphi}_{1} \dot{\varphi}_{2} \\
-m_{2} a_{2} S_{2}\left(\dot{\varphi}_{1}+\dot{\varphi}_{2}\right) \dot{\varphi}_{1} \\
-m_{2} a_{2} C_{2}\left(\dot{\varphi}_{1}+\dot{\varphi}_{2}\right) \dot{\varphi}_{1}
\end{array}\right\|
$$

To determine reaction forces $R_{u}$ (perpendicular with OA) and $R_{v}$ (along OA) at joint $\mathbf{A}$, one uses (18) and (19). For this case, $\mathbf{A}_{1}, \mathbf{A}_{2}$, and $\mathbf{A}_{3}$ are $2 \times 2$ matrices. Equations (18) and (19) have the forms

$$
\left\|\begin{array}{l}
R_{u} \\
R_{v}
\end{array}\right\|=\left\|\begin{array}{c}
-m_{2} g S_{1}+m_{2} a_{2} S_{2}\left(\dot{\varphi}_{1}+\dot{\varphi}_{2}\right)^{2} \\
m_{2} g C_{1}+m_{2} L_{1} \dot{\varphi}_{1}^{2}+m_{2} a_{2} C_{2}\left(\dot{\varphi}_{1}+\dot{\varphi}_{2}\right)^{2}
\end{array}\right\|+\left\|\begin{array}{cc}
m_{2}\left(L_{1}+a_{2} C_{2}\right) & m_{2} a_{2} C_{2} \\
-m_{2} a_{2} C_{2} & -m_{2} a_{2} S_{2}
\end{array}\right\|\left\|\begin{array}{l}
\ddot{\varphi}_{1} \\
\ddot{\varphi}_{2}
\end{array}\right\|,
$$

where $\ddot{\varphi}_{1}$, and $\ddot{\varphi}_{2}$ are calculated from the following equation

$$
\begin{gathered}
\left\|\begin{array}{cc}
m_{1} L_{1}^{2}+a_{2}^{2}+2 L_{1} a_{2} C_{1} & m_{2}\left(L_{1} C_{2}+a_{2}\right) a_{2} \\
m_{2}\left(L_{1} C_{2}+a_{2}\right) a_{2} & m_{2} a_{2}^{2}+J_{2}
\end{array}\right\|\left\|\begin{array}{c}
\ddot{\varphi}_{1} \\
\ddot{\varphi}_{2}
\end{array}\right\| \\
=\left\|\begin{array}{c}
-m_{1} g a_{1} S_{1}-m_{2} g\left(L_{1} S_{1}+a_{2} S_{12}\right)-m_{2} a_{2} L_{1}\left(2 \dot{\varphi}_{1}+\varphi_{2}\right) \dot{\varphi}_{1} \\
-m_{2} g a_{2} S_{12}-m_{2} a_{2} L_{1} S_{2} \dot{\varphi}_{1}^{2}
\end{array}\right\| .
\end{gathered}
$$

It is easy to see that the above example is of the problem of determining the internal forces at the cross section located at the distance of $\mathrm{L} 1$ with respect to the point $\mathrm{O}$, where the reaction forces corresponding to (25) are the tension and shear forces. For the aim of computing the bending moment at the cross section under consideration, it is necessary 
to introduce the constraint equation of the form $f \equiv \varphi_{2}=0$. The reaction force of this constraint is just the bending moment at the cross section of interest.

\subsection{Example 2}

Assume that the non slipping homogeneous disk of radius $r$ and of mass $m$ in Fig. 2 rolls inside the circular cylindrical surface of radius $R$ of a box of mass $M$. The box slides on the perfectly smooth horizontal floor under the influence of force $F$ parallel with the floor and pointed to the right side. Determine the reaction forces between the disk and the box.

The order of freedom of the system is equal to two. The generalized coordinates are chosen as $x, \varphi_{1}$, and $\varphi_{2}$, where $x$ is coordinate of the box's center of mass along the horizontal direction. $\varphi_{1}$, and $\varphi_{2}$ are the angles between $\mathrm{OC}$ and the vertical direction and a radius line $\mathrm{CA}$, respectively. Obviously, the generalized coordinates are dependent. The system's constraint is given by

$$
f \equiv R \dot{\varphi}_{1}+r \dot{\varphi}_{2}=0 .
$$

The kinetic energy of the system can be displayed as (2), where

$$
\mathbf{A}=\left\|\begin{array}{ccc}
m_{0} & m(R-r) C_{1} & 0 \\
m(R-r) C_{1} & 0.5 m r^{2}+m(R-r)^{2} & 0.5 m r^{2} \\
0 & 0.5 m r^{2} & 0.5 m r^{2}
\end{array}\right\| .
$$

The matrix $\mathbf{Q}$ of generalized forces corresponding to the generalized coordinates $\mathrm{x}, \varphi_{1}$, and $\varphi_{2}$ can be derived as

$$
\mathbf{Q}=\left\|\begin{array}{c}
F \\
-m g(R-r) S_{1} \\
0
\end{array}\right\|
$$

Applying a new set of generalized coordinates $x, \varphi_{1}$ and $s$ into the system

$$
s=R \varphi_{1}+r \varphi_{2}
$$

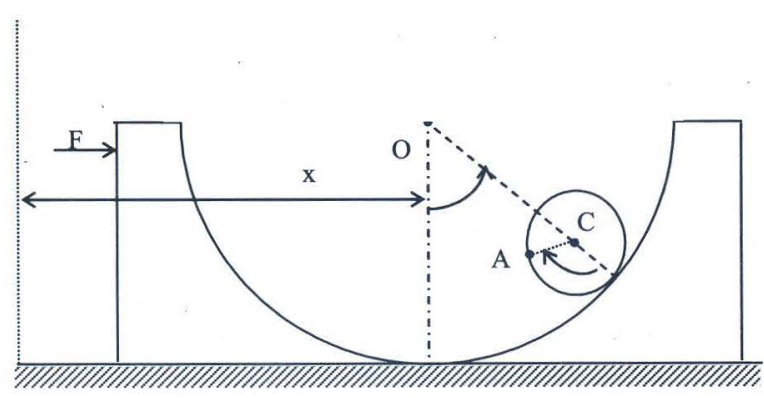

Fig. 2. Example 2 
The transformation matrix $\mathbf{K}$ is given as

$$
K=\left\|\begin{array}{ccc}
1 & 0 & 0 \\
0 & 1 & 0 \\
0 & -\frac{R}{r} & \frac{1}{r}
\end{array}\right\|
$$

The new matrix of inertia can be derived as

$$
\overline{\mathbf{A}}=\mathbf{K}^{\mathbf{T}} \mathbf{A} \mathbf{K}=\left\|\begin{array}{ccc}
m_{0} & m(R-r) C & 0 \\
m(R-r) C & 1.5 m(R-r)^{2} & -0.5 m(R-r) \\
0 & -0.5 m(R-r) & 0.5 m
\end{array}\right\| .
$$

Based on matrix $\overline{\mathbf{A}}$, other matrices such as $\overline{\mathbf{Q}}, \overline{\mathbf{Q}}^{\mathbf{0}}$, and $\overline{\mathbf{Q}}^{*}$ can be derived as

$$
\begin{aligned}
& \overline{\mathbf{Q}}=\mathbf{K}^{\mathbf{T}} \mathbf{Q}=\left\|\begin{array}{ccc}
1 & 0 & 0 \\
0 & 1 & -\frac{R}{r} \\
0 & 0 & \frac{1}{r}
\end{array}\right\| \begin{array}{c}
F \\
-m g(R-r) S_{1} \\
0
\end{array}\|=\| \begin{array}{c}
F \\
-m g(R-r) S_{1} \\
0
\end{array} \| \\
& \mathbf{Q}^{\mathbf{0}}=\left\|\begin{array}{c}
0 \\
-m(R-r) S_{1} \dot{x} \dot{\varphi} \\
0
\end{array}\right\| ; \quad \mathbf{Q}^{*}=\left\|\begin{array}{c}
-m(R-r) S_{1} \dot{\varphi}_{1}^{2} \\
-m(R-r) S_{1} \dot{x} \dot{\varphi}_{1} \\
0
\end{array}\right\| \text {. }
\end{aligned}
$$

In the new coordinate system, the constraint now has the form

$$
s=0 \text {. }
$$

Based on the ideality of constraints, matrix $\mathbf{D}$ is given as

$$
\mathbf{D}=\left\|\begin{array}{lll}
1 & 0 & 0 \\
0 & 1 & 0
\end{array}\right\|
$$

From (15), we have

$$
R_{x}=0 ; \quad R_{\varphi_{1}}=0 .
$$

Note that matrix $\mathbf{A}_{1}$ is of $2 \times 2$ size

$$
\mathbf{A}_{1}=\left\|\begin{array}{cc}
m_{0} & m(R-r) C_{1} \\
m(R-r) C_{1} & 1.5 m(R-r)^{2}
\end{array}\right\| ;
$$

$\mathbf{A}_{3}$ and $\mathbf{A}_{2}$ are matrices of $2 \times 1$ size and $1 \times 1$ size, respectively:

$$
\begin{aligned}
& \mathbf{A}_{2}=\|0.5 m\| ; \mathbf{A}_{3}^{T}=\|0-0.5 m(R-r)\| \\
& R_{s}=Q_{s}+a_{x s} \ddot{x}+a_{\varphi s} \ddot{\varphi}_{1}=-0.5 m(R-r) \ddot{\varphi}_{1}
\end{aligned}
$$

where $\ddot{\varphi}_{1}$ is calculated through (19) as

$$
\begin{aligned}
& m_{0} \ddot{x}+m(R-r) C_{1} \ddot{\varphi}_{1}=m(R-r) S_{1} \dot{\varphi}_{1}^{2}+F \\
& m(R-r) C_{1} \ddot{x}+1.5 m(R-r)^{2} \ddot{\varphi}_{1}=-m g(R-r) S_{1}
\end{aligned}
$$

From (44), and (45), the reaction force $R_{s}$ is derived as

$$
R_{s}=-\frac{0.5 m}{m C_{1}^{2}}\left[m(R-r) C_{1} S_{1} \dot{\varphi}^{2}\right]+F C_{1}+m g S_{1} .
$$




\section{CONCLUSIONS}

In the paper, a new approach of determining reaction forces of planar mechanisms is presented. The approach can be applied to dynamics and endurance analysis of machines. The method can also be used for the problems of stability and control. The advantage of the method is matrix-based so that complicated problems can be solved easily by using such software as Mathlab, Maple, and Mathcad.

\section{ACKNOWLEDGMENT}

The publication is completed thank to the financial support from the National Basics Research Program in Natural Sciences.

\section{REFERENCES}

[1] Do Sanh, On the Principle of Compatibility and the Equations of Motion of Constrained Mechanical System, ZAMM 60 (1980) 210-212, Kleine Miteilungen.

[2] Do Sanh, On the motion of controlled mechanical systems, Advance in Mechanics, Tom 7, Vol.2, Varsaw, 1984

[3] Do Sanh, Dinh Van Phong, Do Dang Khoa, Realization of program motion in asymptotical sense, The $5^{\text {th }}$ Asian Symposium on Applied Electromagnetics and Mechanics, Science and Technology Publishing House, pp 421-430, Hanoi, 2005.

[4] Do Sanh, Do Dang Khoa, The method of transmission matrix for investigating planar mechanisms, Vietnam Journal of Mechanics 29 (1) (2007) 37-46.

[5] Do Sanh, Do Dang Khoa, Method of transmission matrix for investigating planar relative motions, Vietnam Journal of Mechanics 29 (2) (2007) 105-11 6.

[6] Do Sanh, Do DangKhoa, Method of calculating the inertia matrix in the problems of dynamics of planar mechanisms, The $3^{\text {th }}$ Vietnam Conference on Mechatronics, pp.357-362, Hanoi 2006 (in Vietnamese).

[7] A. I. Lurie, Analytical Mechanics, Fizmatgiz. Publ. Moscow, 1961 (in russian)

Received August 20, 2008

\section{XÁC ĐỊNH PHẢN LỰC TRONG CƠ CẤU PHẲNG}

Trong bài báo đề xuất một phương pháp xác định phản lực trong cơ cấu phẳng và trạng thái nội lực (động lực) tại các mặt cắt của các khâu. Ý tưởng của phương pháp dựa trên nguyên lý phù hợp với việc tạo ra những liên kết mới và điều kiện của liên kết lý tưởng. 